\title{
In situ and real-time 3-D microtomography investigation of dendritic solidification in an $\mathrm{Al}-10 \mathrm{wt} . \% \mathrm{Cu}$ alloy
}

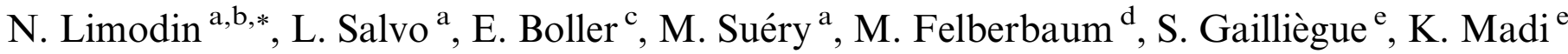 \\ ${ }^{a}$ SIMAP, UMR CNRS 5266, Grenoble INP, UJF, GPM2, BP 46, 38402 Saint-Martin d'Hères Cedex, France \\ ${ }^{\mathrm{b}}$ MATEIS, UMR CNRS 5510, INSA de Lyon, 69621 Villeurbanne Cedex, France \\ ${ }^{\mathrm{c}}$ ESRF, 156 rue des Martyrs, BP 220, 38043 Grenoble Cedex 9, France \\ ${ }^{\mathrm{d}}$ Laboratoire de Simulation des Matériaux, Ecole Polytechnique Fédérale de Lausanne, CH-1015 Lausanne, Switzerland \\ ${ }^{\mathrm{e}}$ Mines Paris, ParisTech, Centre des Matériaux/UMR CNRS 7633, BP 87, 91003 Evry Cedex, France
}

Received 4 April 2008; received in revised form 21 January 2009; accepted 28 January 2009

Available online 4 March 2009

\begin{abstract}
The microstructural evolution of an $\mathrm{Al}-10 \mathrm{wt} . \% \mathrm{Cu}$ alloy was investigated during solidification at constant cooling rate by in situ synchrotron X-ray microtomography with a resolution of $2.8 \mu \mathrm{m}$. Solidification of this alloy leads to a coarse dendritic microstructure which was fully characterized in terms of variation with temperature of the solid fraction, the specific surface area of the solid-liquid interface and the local curvatures of the solid phase. By analysing the evolution with solid fraction of individual dendrites, at least two coarsening mechanisms were clearly identified in addition to solidification growth. The first mechanism involves remelting of small secondary dendrite arms to the benefit of bigger adjacent arms. The second is the coalescence of adjacent secondary arms, with progressive filling of the inter-arm spacing and coalescence at the tips. Although this mechanism preferentially occurs at high solid fractions, these results show that the evolution of the dendritic microstructure during solidification is complex and involves the occurrence of various mechanisms operating concurrently. In situ X-ray tomography thus allows revisiting the various models which have been proposed to account for dendrite coarsening during solidification.
\end{abstract}

(C) 2009 Acta Materialia Inc. Published by Elsevier Ltd. All rights reserved.

Keywords: Semi-solid; 3-D X-ray tomography; Aluminium alloys; Dendritic solidification; Coarsening

\section{Introduction}

Solidification of metallic alloys in industrial casting processes involves the formation of solid dendrites, the characteristics of which depend on the cooling rate in particular. At high cooling rates, the dendrites are well defined, with primary, secondary and sometimes tertiary dendrite arms. At low cooling rates, conversely, the dendrites evolve strongly during cooling by diffusion of solute in the liquid so that the dendritic structure becomes degenerated. This

\footnotetext{
${ }^{*}$ Corresponding author. Address: MATEIS, UMR CNRS 5510, INSA de Lyon, 69621 Villeurbanne Cedex, France. Tel.: +33 4724371 76; fax: +33472438539.

E-mail address: nathalie.limodin@insa-lyon.fr (N. Limodin).
}

evolution is even more pronounced when the alloy is held isothermally in the semi-solid range, sometimes leading to globular microstructures suitable for thixoforming.

Based on Kahlweit's observations of organic materials, used as transparent analogue systems for metallic alloys, Kattamis et al. proposed two models that can predict the evolution of microstructural parameters such as the specific surface area of the solid-liquid interface, $S_{\mathrm{v}}$, during partial remelting [1] and during solidification [2-4]. "Model A", illustrated in Fig. 1a, describes the remelting of a small dendrite arm from its tip to its root to the benefit of bigger adjacent arms; the small arm is assumed to shrink at constant radius while the larger arms grow in radii at constant length. Diffusion of matter is supposed to occur through the liquid. Indeed, according to the Gibbs-Thomson 
a

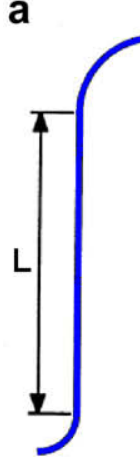

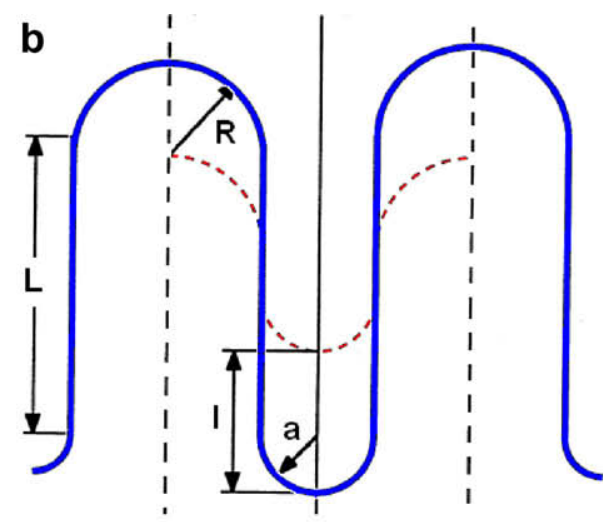
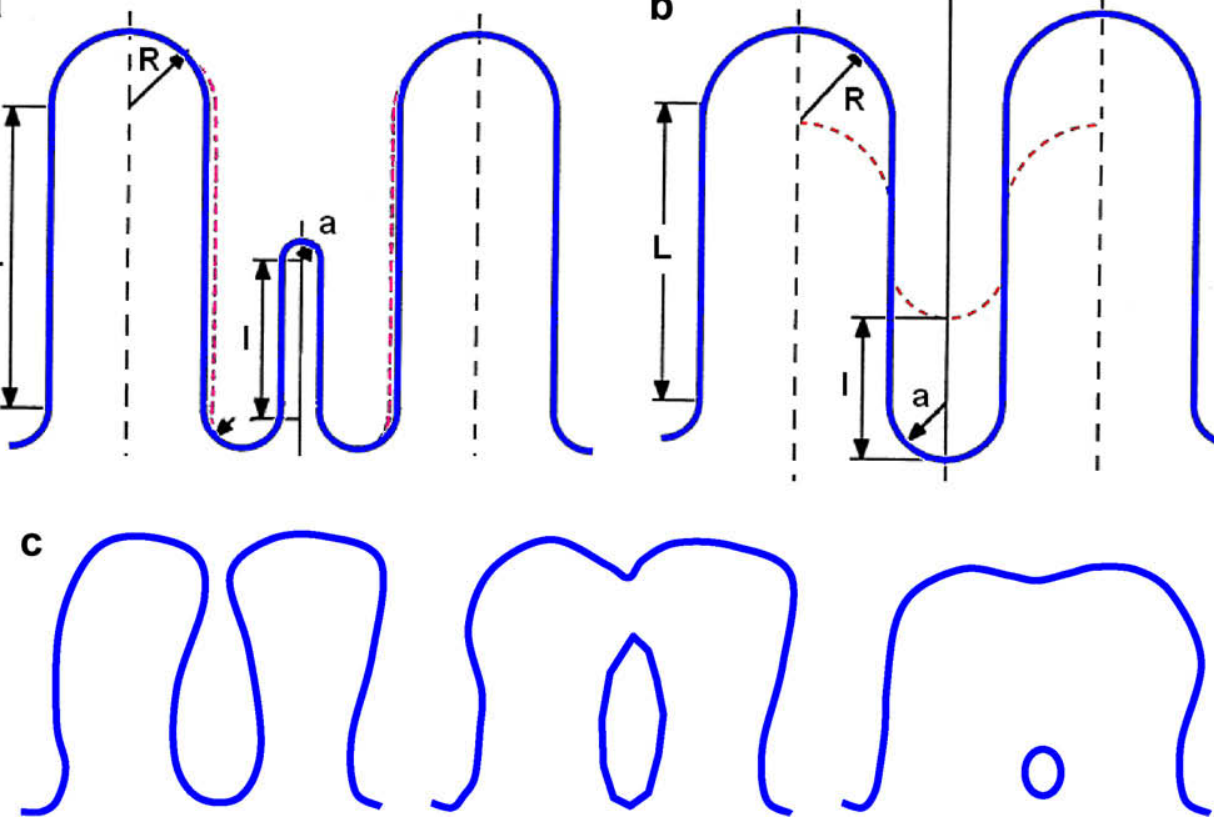

Fig. 1. Diagrams of (a) model A and (b) model B, reprinted from Ref. [4]; and of (c) the coalescence mechanism proposed by Mortensen [6].

equation, the solute concentration in the liquid at a liquidsolid interface is larger for regions with large curvature than for regions with small curvature. Thus, a flow of mass establishes from areas of large curvature towards those of small curvature. "Model B", illustrated in Fig. 1b, describes the coalescence of adjacent arms with remelting of the tips and deposition at the root between them. These models were shown to describe the evolution of the specific surface area of the solid-liquid interface quite well when applied to post-quench experiments, particularly for short holding times. Young and Kirkwood [5], and later Mortensen [6], also proposed several models to describe coalescence of adjacent dendrite arms. A coalescence mechanism reported by Mortensen is illustrated in Fig. 1c: the dendrite arms have tear shapes, which may result from dissolution of the dendrite arm at the root and redeposition at the tip [5], and they coalesce at their tips with a liquid pool that eventually forms at the root.

Usually, the microstructures of metallic alloys are studied on metallographic sections obtained after complete solidification of the specimen or by quenching from the semi-solid state. Recently, the development of synchrotron techniques has allowed in situ observations of the evolution of microstructure during solidification. In particular, microradiography has been extensively used to study the solidification of alloys such as $\mathrm{Al}-\mathrm{Ni}$ [7], $\mathrm{Al}-\mathrm{Cu}$ [8] and $\mathrm{Sn}-\mathrm{Bi}$ [9] in real-time. Radiography permits observation of the mechanisms at work at a time interval as low as $0.15 \mathrm{~s}$ [8]. This small time resolution allows visualization of the first stages of solidification inside samples thin enough (100-200 $\mu \mathrm{m}$ thick) for a single layer of dendrites to be observed. For example, Reinhart et al. [7] were able to observe and identify the interactions that occur between dendrites during the columnar to equiaxed transition
(CET) using a large field of view, e.g. $15 \times 15 \mathrm{~mm}^{2}$, inside the sample. This allows them better understanding the factors, such as the pulling rate, that influence the CET [7]. However, a large field of view could only be obtained to the detriment of the spatial resolution, i.e. about $7-10 \mu \mathrm{m}$. Thus, the fineness of the microstructure, combined with a low absorption contrast between the solid and liquid phases at the beginning of solidification, make quantitative analysis on the scale of the dendrite arms difficult. Reducing the field of view to $1.5 \times 1.5 \mathrm{~mm}^{2}$ allows reaching a spatial resolution of $1.5 \mu \mathrm{m}$. For example, this has been used to obtain quantitative information about the solute concentration field in the liquid to understand how fragmentation of dendrites occurs $[8,10]$. In situ microradiography has mainly been applied to directional solidification conditions, i.e. with a temperature gradient, except for a study by Li et al. [9] in which the solidification conditions involved no temperature gradient. Real-time observations of dendritic semi-solid Sn-Bi using synchrotron microradiography permit qualitative observations of the mechanisms that operate on the dendritic microstructure, such as coalescence and dendrite arm remelting from tip towards root [9]. It was reported that coarsening by the remelting of the small dendrite arms prevails over coalescence, although both are observed at slow cooling rates, and that coarsening primarily affects the dendritic morphology during the early stages of solidification, i.e. $15-25 \%$ of the total solidification time [9].

The aim of the present study was to observe, in real-time and in three dimensions, the microstructural evolution of an $\mathrm{Al}-\mathrm{Cu}$ alloy during solidification with no temperature gradient using fast X-ray microtomography in order to gain a better understanding of the dendrite arm interactions and to assess the validity of existing models. 


\section{Experimental methods}

\subsection{Material and X-ray microtomography device}

An $\mathrm{Al}-10$ wt.\% $\mathrm{Cu}$ alloy was chosen because the solid and liquid phases have $\mathrm{Cu}$ contents sufficiently different to provide good absorption contrast. The eutectic and liquidus temperatures of the alloy are 548.2 and $634^{\circ} \mathrm{C}$, respectively. The alloy was prepared as follows: copper (99.99\% pure, Oxygen Free Hard Copper) and aluminium $(99.99 \%)$ were put in a crucible under a controlled atmosphere which was flushed first with argon to obtain $2 \times 10^{-5}$ mbar, then with forming gas $\left(\mathrm{N}_{2}-10\right.$ vol. $\left.\% \mathrm{H}_{2}\right)$. The mixture was melted and mixed at $833^{\circ} \mathrm{C}$ before casting in a copper mould coated with boron nitride. A cylindrical specimen of about $1.4 \mathrm{~mm}$ diameter was machined from this ingot.

The tomography experimental set-up is illustrated in Fig. 2. The specimen was glued with zirconia paste on top of an alumina rod placed on the rotating stage. The furnace is made of two $\mathrm{MoSi}_{2}$ heaters enclosed inside a cubic-shaped chamber. It has a hole at the bottom through which the specimen is inserted and two windows on the sides to allow the X-rays through. The sample was supported by its own oxide skin up to the temperature of the experiment. The specimen was gradually heated to a temperature slightly above the liquidus. It was maintained in the liquid state for a few minutes. Next, the specimen was cooled at a controlled rate of $-3{ }^{\circ} \mathrm{C} \mathrm{min}^{-1}$ while $\mathrm{X}$-rays were switched on and the microtomography experiment was carried out; a low cooling rate was necessary to ensure that the microstructure did not evolve too much during the image acquisition.

The experiments were conducted on the ID19 beam line at the ESRF. An almost monochromatic X-ray beam $(\Delta \lambda)$ $\lambda \approx 0.01$ ) is used and a high-speed camera records the transmitted intensity while the sample is continuously rotated over $180^{\circ}$. Four hundred and fifty projections were taken during each $180^{\circ}$ rotation. Dark field images, i.e. without X-rays, and flat field images, i.e. with X-rays but without the sample, were also recorded for further processing of the images. Due to the furnace dimensions, the distance between the specimen and the camera was $90 \mathrm{~mm}$. This is small enough to reduce the phase contrast [11] that is detrimental to further quantitative analysis of the tomographic images. A FReLoN (FastReadoutLowNoise) 14-bit dynamic CCD camera with a $2048 \times 2048$ pixel chip was used. The camera pixel size of $14 \mu \mathrm{m}$ is reduced to $1.4 \mu \mathrm{m}$ with the appropriate optic system. The readout time of the camera is about $50 \mathrm{~ms}$ in full frame mode. To minimize the total scan time and avoid image blurring due to evolution with time of the semi-solid microstructure, this readout time has to be reduced. Therefore, the camera was used in frame transfer mode (FTM). In FTM, the $2048 \times 2048$ pixel chip of the CCD camera is divided in three horizontal parts: a photosensitive zone in the centre of the camera and a memory zone on both sides of the photosensitive zone. The size of the image is thus reduced to $2048 \times 1024$ pixels, i.e. the size of the photosensitive zone, which is further decreased to $1024 \times 1024$ pixels by defining a (vertical) region of interest. The frame transfer mode allows the reading of a frame in the CCD memory zone while exposing the photosensitive zone to acquire the following image. To further reduce the scan time, the camera was used in binning mode, i.e. the content of four adjacent pixels is added. Finally, the projection size is $512 \times 512$ pixels and the scan time for a full rotation is about $22 \mathrm{~s}$, which is assumed to be less than the time required for significant microstructural changes to occur. The resulting pixel size is $2.8 \mu \mathrm{m}$. A scan was taken every minute to characterize the microstructural evolution during solidification. The total solidification time was about 1800 s.

\subsection{Image processing}

The method for reconstruction of the volumes from the projections has been detailed elsewhere [12]. After reconstruction, the images are cropped to a size of $260 \times$ $254 \times 200$ pixels, i.e. $0.73 \times 0.71 \times 0.56 \mathrm{~mm}$, in order to be able to perform three-dimensional (3-D) calculations with a conventional computer. The same set of images was also cropped with a larger cross-section but smaller height, i.e. $318 \times 342 \times 58$, in order to focus on a given dendrite, which was artificially separated from its neighbours for further analysis. In both cases, a 3-D regiongrowing algorithm was used to segment the solid and liquid phases in the greyscale image. The reader is referred to Ref. [13] for details about image processing.

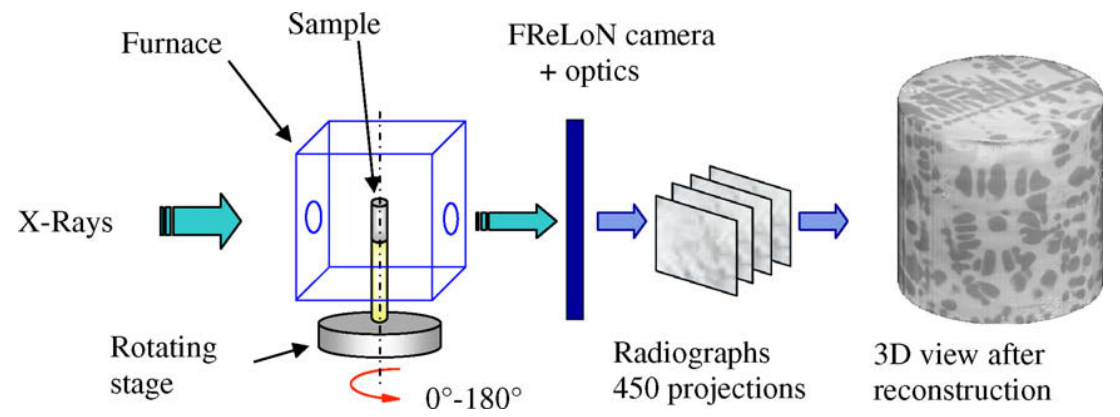

Fig. 2. Schematic diagram of experimental set-up. 


\section{Results and discussion}

\subsection{Analysis of dendritic solidification on the sample scale}

\subsubsection{Qualitative analysis}

Images of the partially solidified microstructure with sufficient contrast between the solid and liquid phases were obtained for volume fractions of solid larger than 0.2. In the beginning of solidification, the nucleated equiaxed dendrites are free to move in the liquid. The density of the solid is less than that of the copper-rich liquid [14]; the dendrites therefore tend to float and the time resolution does not allow a proper image to be captured for image analysis. As soon as the growing dendrites become entangled in the volume of the specimen and a coherent solid network is formed, it is possible to have a clear image of the microstructure. Furthermore, the absorption contrast increases with solidification time as the liquid phase is enriched in solute faster than the solid phase.

Two-dimensional slices were extracted from a given cross-section of the specimen for different solidification times and are shown in Fig. 3. No temperature gradient is applied to the specimen so that dendrites may nucleate anywhere in the specimen and they are free to grow in any direction. However, most of the dendrites were observed to lie parallel to the cross-section of the sample, as in Fig. 3. Dendrites probably nucleate on the oxide skin on the specimen surface and grow radially towards the sample centre. In the image acquired at $107 \mathrm{~s}$ of solidification, the contrast between the liquid and solid phases is not good enough to allow segmentation of the two phases and subsequent image analysis. Thus, only qualitative information could be obtained. If this image is compared to the one obtained at $163 \mathrm{~s}$ of solidification, we can observe that the primary trunk shows a zone free of any side-branches at $163 \mathrm{~s}$ in a region where dendrite arms were observed some $56 \mathrm{~s}$ earlier. This could result from fragmentation of the dendrite that occurs by detachment of secondary dendrite arms at their roots as reported in [10]. However, as soon as a coherent solid network is formed, fragmentation no longer seems to be favoured. The figure shows clearly that the microstructure changes drastically in the first stages of solidification. From about $800 \mathrm{~s}$ of solidification and onwards, the microstructure evolves much more slowly

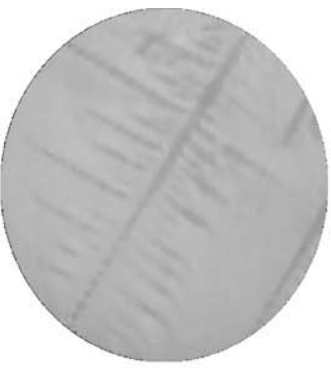

$107 \mathrm{~s}, 629^{\circ} \mathrm{C}$

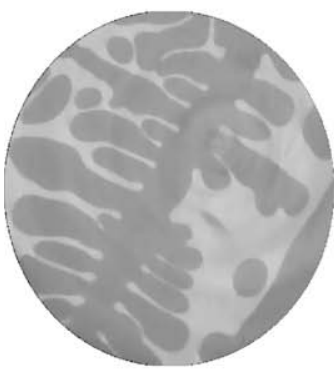

$440 s, 612^{\circ} \mathrm{C}$

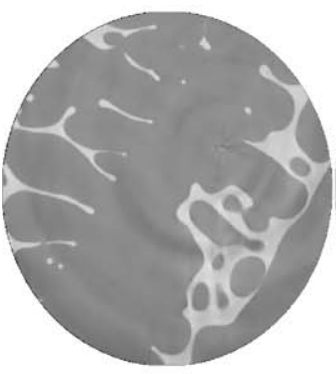

$1104 \mathrm{~s}, 579^{\circ} \mathrm{C}$

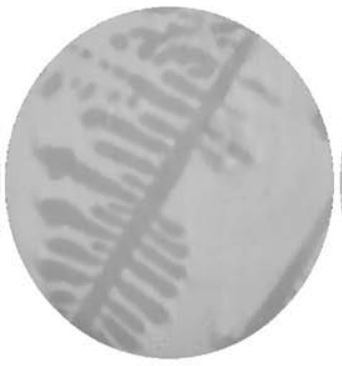

$163 \mathrm{~s}, 6^{\circ} \mathrm{C}$

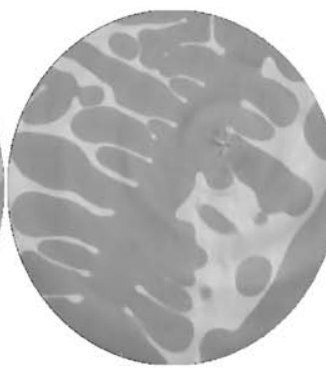

$603 s, 604^{\circ} \mathrm{C}$

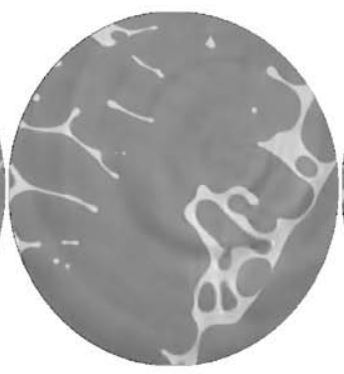

1270 s, $571{ }^{\circ} \mathrm{C}$

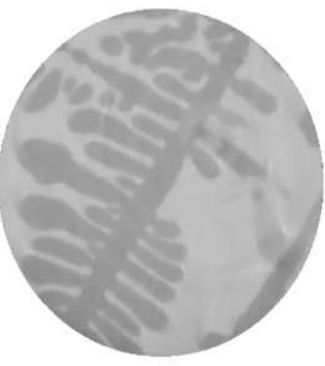

$219 s, 623^{\circ} \mathrm{C}$

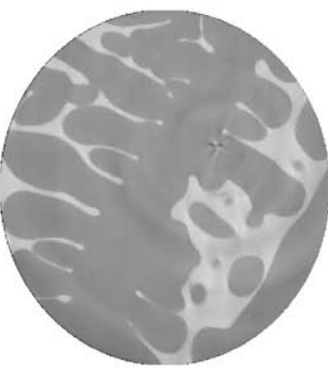

$768 s, 596^{\circ} \mathrm{C}$

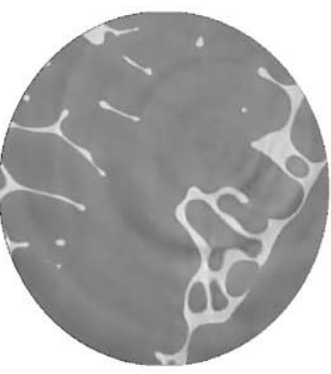

$1439 \mathrm{~s}, 562^{\circ} \mathrm{C}$

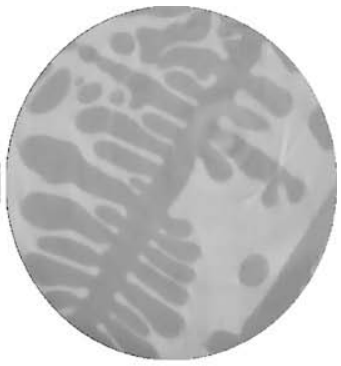

$275 s, 620^{\circ} \mathrm{C}$

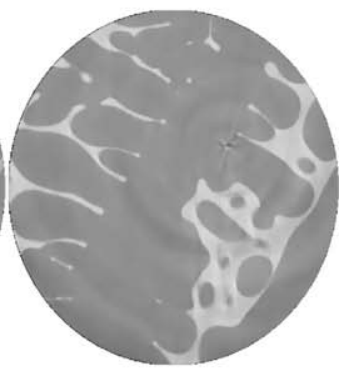

$935 \mathrm{~s}, 587^{\circ} \mathrm{C}$

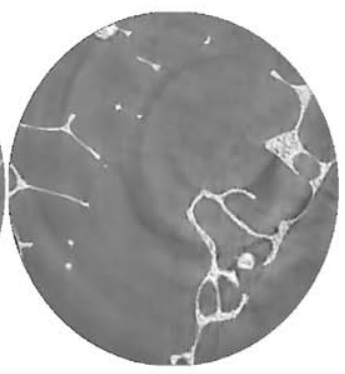

$1933 \mathrm{~s}, 537^{\circ} \mathrm{C} \quad\left(<T_{E}\right)$

Fig. 3. Evolution with time as shown by a series of 2-D slices extracted from the bulk specimen. 


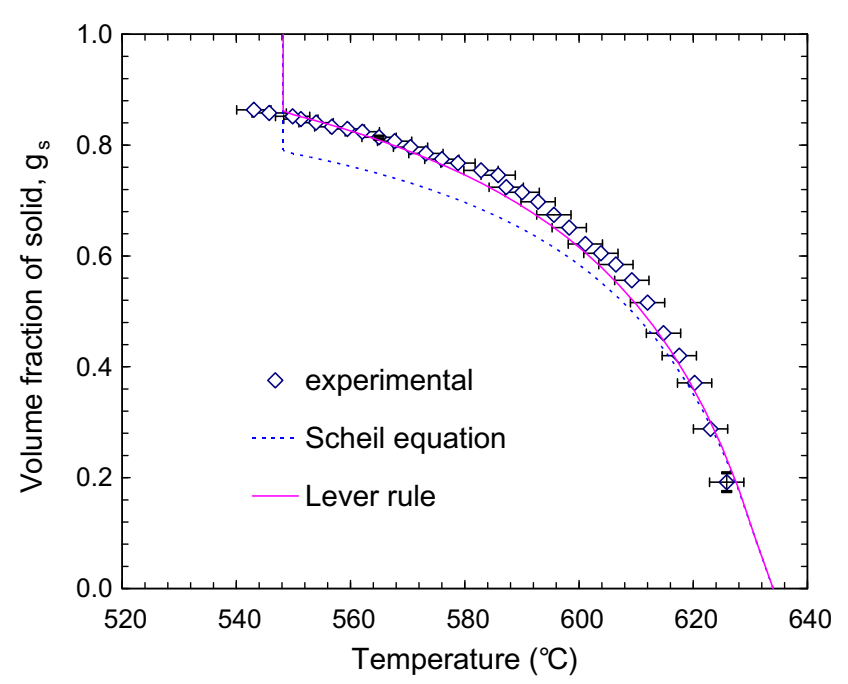

Fig. 4. Variation of the solid fraction with temperature (error bars on temperature and solid fraction measurement added).

until solidification is complete and eutectic is formed in the interdendritic space, i.e. at 1933s in Fig. 3.

\subsubsection{Quantitative analysis}

As soon as the solid-liquid interface is resolvable, image processing can be performed and quantitative information obtained.

Fig. 4 shows the variation of volume fraction of solid with temperature obtained from 3-D analysis. The experimental results are roughly in agreement with solid fractions predicted by the lever rule or by the Scheil equation. However, they are closer to and sometimes higher than the lever rule prediction. On the one hand, this can be attributed to the very low cooling rate that generates conditions close to thermodynamic equilibrium. On the other hand, uncertainty in measurements of the specimen temperature and the solid fraction and in the alloy composition could affect the curves in Fig. 4. The eutectic is also observed to form about $8{ }^{\circ} \mathrm{C}$ below the theoretical eutectic temperature; this is most probably due to temperature uncertainty. The temperature of the specimen was calibrated assuming that the first tomographic scan where solid is observed corresponds to the liquidus temperature and that the specimen is cooled at a constant cooling rate of $3{ }^{\circ} \mathrm{C} \mathrm{min}^{-1}$, i.e. equal to the cooling rate imposed by the furnace. Therefore, the initial error on the temperature is $\pm 3{ }^{\circ} \mathrm{C}$, i.e. the temperature interval between consecutive scans; this can increase with solidification time if the specimen temperature does not cool at the same rate as the furnace. The error in the solid fraction is mainly due to the error from segmentation, which was calculated using the minimum and maximum acceptable threshold values used to segment the liquid and solid phases. As expected, the error in the solid fraction, reported in Fig. 4 for two temperatures, is small, especially at high solid fraction, where the absorption contrast is maximum.

The specific surface area of the solid-liquid interface normalized by the total volume of analysis, $S_{\mathrm{v}}^{*}$, was

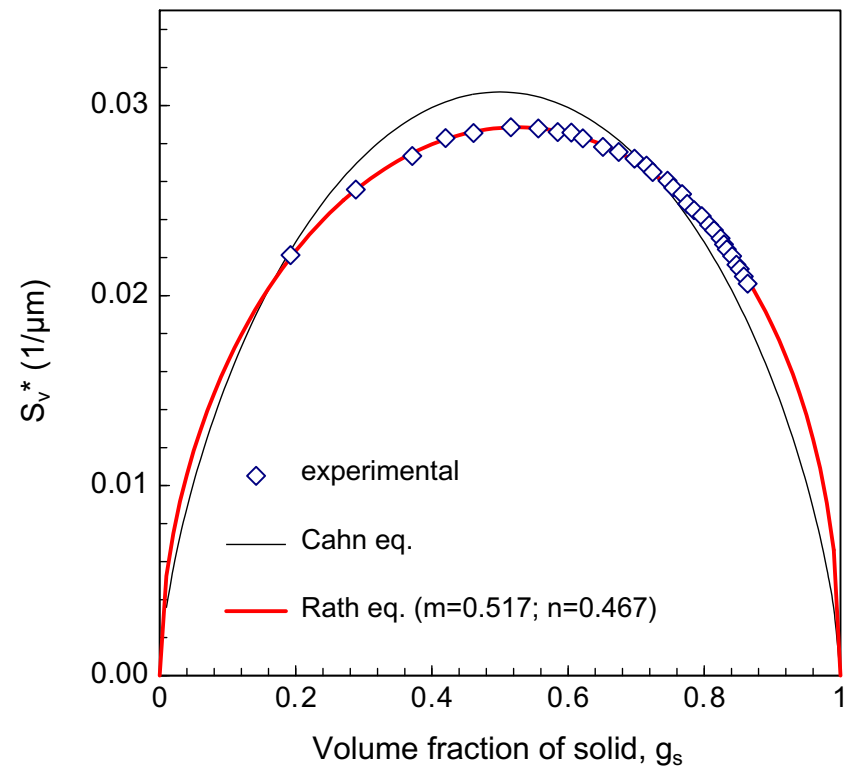

Fig. 5. Variation with solid fraction of the specific surface area of the solid-liquid interface, $S_{\mathrm{v}}^{*}$, normalized by the total volume of analysis.

measured using a "marching cubes" algorithm developed by Lorensen and Cline [15] for 3-D surface construction. The variation with time is shown in Fig. 5. The experimental points are better represented by the relationship suggested by Rath [16] than by the one suggested by Cahn [17]:

$S_{\mathrm{v}}^{*}=K \times\left(g_{\mathrm{s}}\right)^{m} \times\left(1-g_{\mathrm{s}}\right)^{n}$

where $K$ is a constant and $m=n=2 / 3$ according to Cahn or $0<(m, n)<1$ is obtained by fitting the experimental data according to Rath.

The above equation links the specific surface area of the transformed phase to its volume fraction, taking an impingement factor into account. Although they consider nucleation and growth of spheroidal grains in the solid state, their relationships match the evolution of $S_{\mathrm{v}}^{*}$ in dendritic solidification quite well, particularly the Rath equation. Contrary to Cahn, Rath assumes that the rate of nucleation and growth of the newly formed surface (the solid) at small volume fraction should not be equal to the rate of disappearance of the matrix (the liquid) at large volume fraction because the shape of the phases is not the same. However, Ludwig et al. [12] reported that a law that is symmetric with respect to $g_{\mathrm{s}}=0.5$, such as Cahn's equation, fits the evolution of $S_{\mathrm{v}}$ during dendritic solidification with a fully equiaxed microstructure. Therefore, the dissymmetry observed in the present study seems to be related to a more complex dendritic microstructure.

\subsection{Analysis of dendritic solidification at the scale of a single dendrite}

For solid fractions ranging from 0.2 to about 0.7 , it was possible to isolate part of one dendrite, from neighbouring dendrites inside the volume of analysis. This dendrite was shown in the 2-D slices in Fig. 3. Three-dimensional 


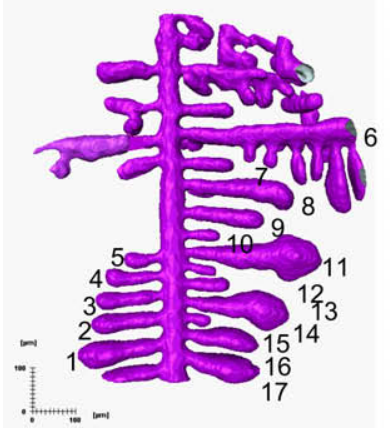

$163 \mathrm{~s}, 626^{\circ} \mathrm{C}, 0.19$

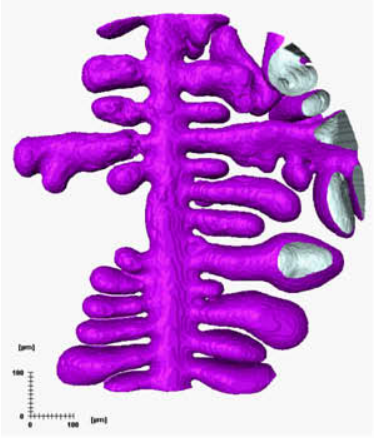

$384 \mathrm{~s}, 615^{\circ} \mathrm{C}, 0.46$

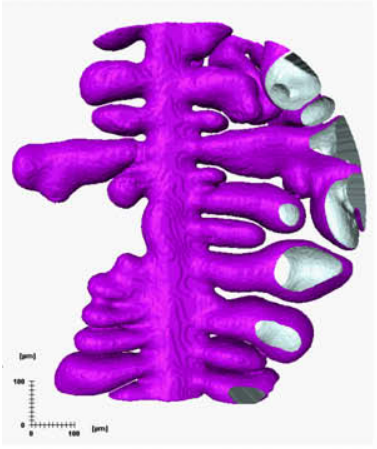

$603 \mathrm{~s}, 604^{\circ} \mathrm{C}, 0.60$

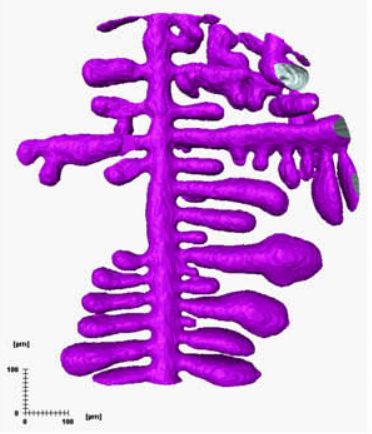

$219 \mathrm{~s}, 623^{\circ} \mathrm{C}, 0.29$

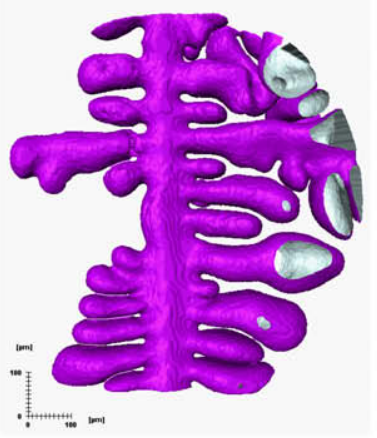

$440 \mathrm{~s}, 612^{\circ} \mathrm{C}, 0.52$

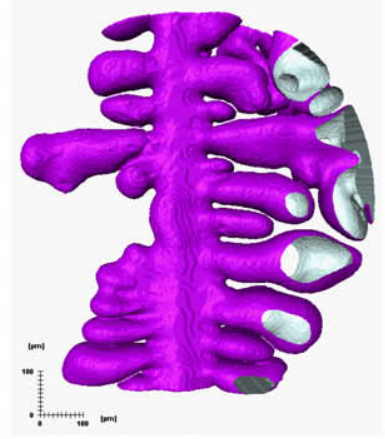

$658 \mathrm{~s}, 601^{\circ} \mathrm{C}, 0.62$

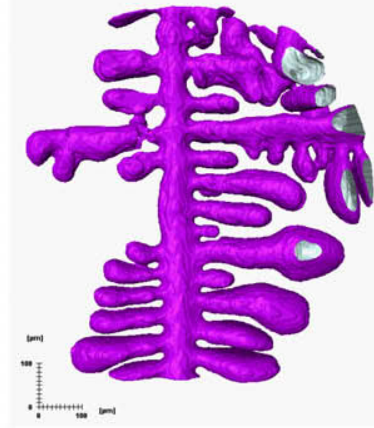

$275 \mathrm{~s}, 620^{\circ} \mathrm{C}, 0.37$

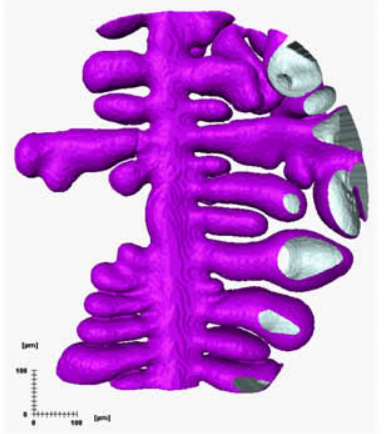

$495 \mathrm{~s}, 609^{\circ} \mathrm{C}, 0.56$

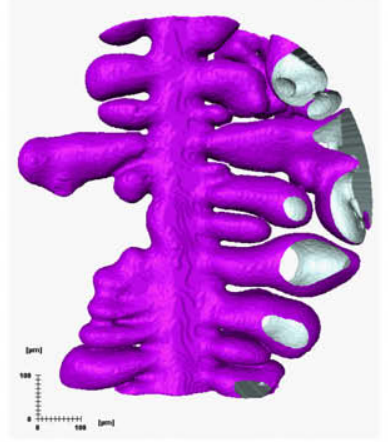

$714 \mathrm{~s}, 598^{\circ} \mathrm{C}, 0.65$

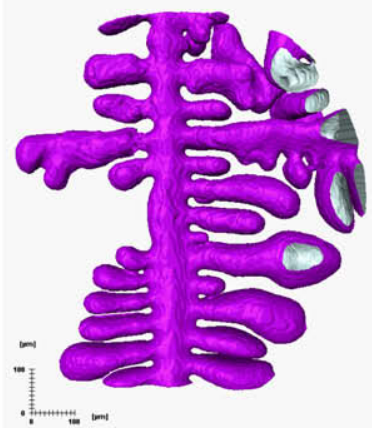

$329 \mathrm{~s}, 618^{\circ} \mathrm{C}, 0.42$

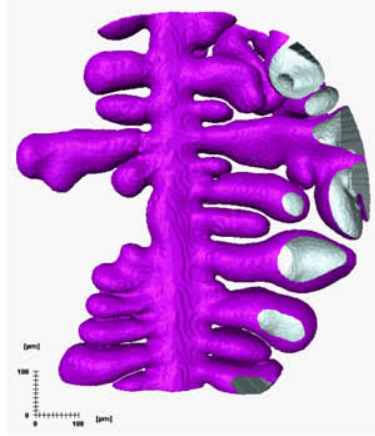

$551 \mathrm{~s}, 606^{\circ} \mathrm{C}, 0.58$

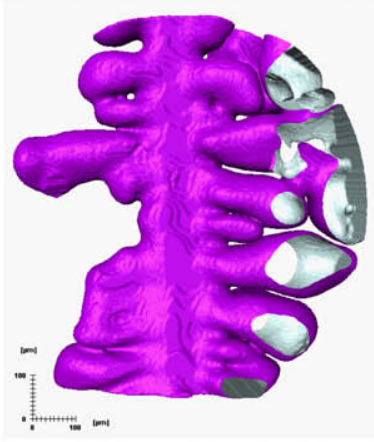

$1104 \mathrm{~s}, 579^{\circ} \mathrm{C}, 0.77$

Fig. 6. Three-dimensional observation of the evolution of a dendrite with solidification time.

observations performed on this dendrite are shown in Fig. 6 for increasing solidification times. It is important to note that the dendrite morphology evolves by both solidification growth (increase of the solid volume fraction) and coarsening mechanisms driven by the reduction of the solid-liquid interface energy, which could be important at low cooling rates.

\subsubsection{Qualitative analysis}

Several coarsening mechanisms are observed to act upon the secondary and tertiary arms of this dendrite during solidification (Fig. 6).

On the left-hand side of the dendrite, the roots between adjacent arms labelled from 1 to 5 are progressively filled with solid and the tips of the adjacent arms grow until they touch and join each other. The filling of the roots and the joining of the arm tips can result from solidification growth of the primary trunk and of the tips of the dendrite arms, respectively. However, this could also result from a coalescence mechanism that resembles the mechanisms proposed by Kattamis et al. (Model B in Fig. 1b) and by Mortensen (Fig. 1c) for coarsening during isothermal holding. Contrary to the assumption made in model $\mathrm{B}$, the dendrite arms are not cylindrical but, rather, tear-shaped, as in Fig. 1c. It is important to note that the distinction between solidification growth and coalescence is very difficult as solidification and coarsening are occurring at the same time.

On the right-hand side of the primary stem, the small secondary arms, e.g. the arms labelled 15, 12 and 10, dissolve from the tips towards the roots to the benefit of larger 
a

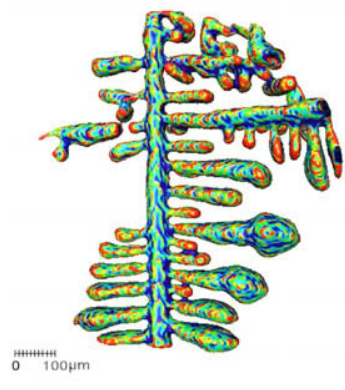

$163 s$

$g_{s}=0.19$

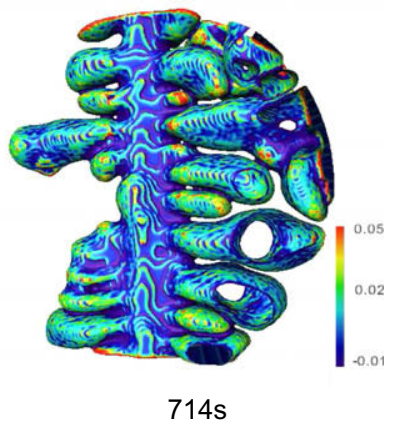

$g_{s}=0.70$

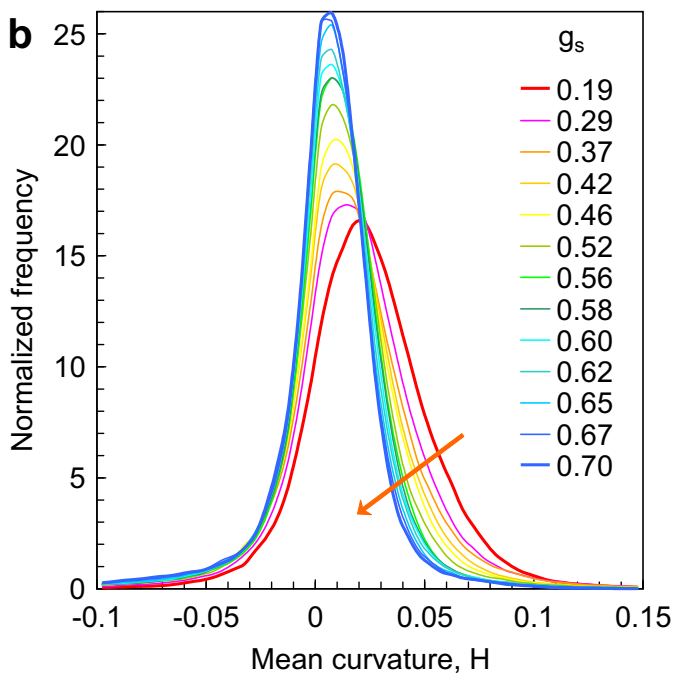

C

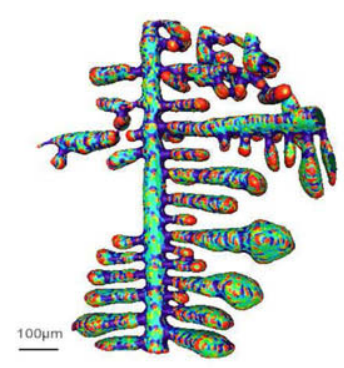

$163 \mathrm{~s}$

$g_{s}=0.19$

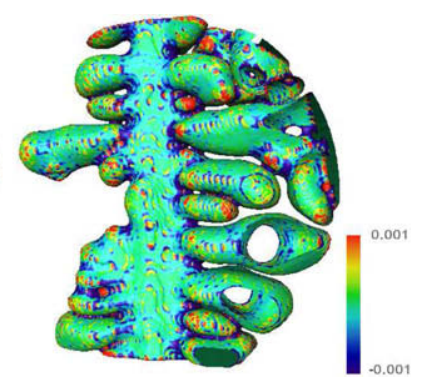

$714 \mathrm{~s}$

$g_{s}=0.70$

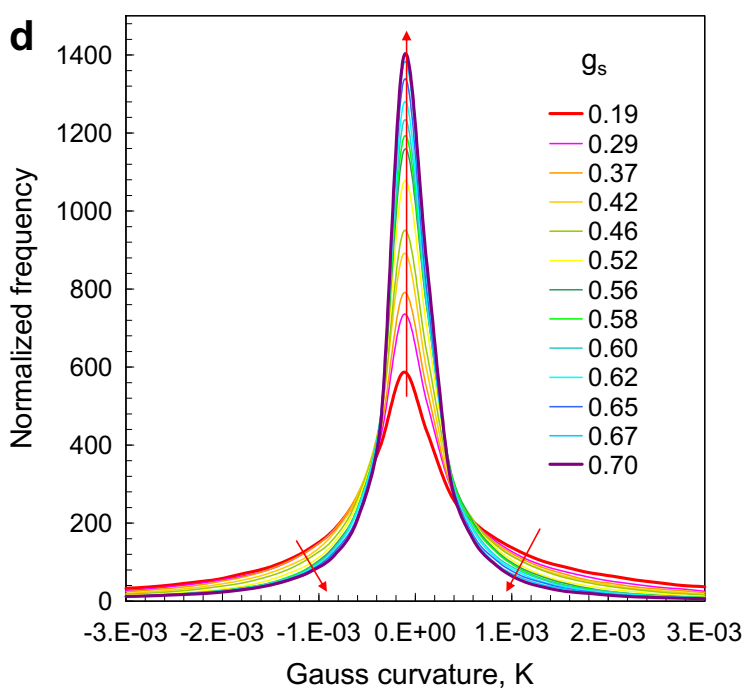

Fig. 7. Mean (a) and Gauss curvature (c) areas at the surface of the dendrite and their distributions with increasing solid fraction ((b) and (d), respectively).

adjacent arms. Solidification growth is unable to account for the dissolution of dendrite arms. Thus, smaller arms on the right-hand side of the dendrite probably dissolve according to a remelting mechanism similar to model A (Fig. 1a) while solidification occurs. Kattamis suggested that coarsening by model B, i.e. coalescence, occurs after coarsening according to model A. However, it is difficult to assess whether coarsening by model A prevails at short times since both mechanisms are observed to operate in the present study. On the secondary arm labelled 6 , we can observe that two tertiary arms close to the primary trunk are coarsening via a mechanism that is very similar to model B: the tips of the adjacent arms do not coalesce and the filling of the root between the arms cannot be ascribed only to the growth of the secondary arm trunk.

\subsubsection{Quantitative analysis}

The mean curvature $H$ is defined as: $H=0.5 \times$ $\left(1 / R_{1}+1 / R_{2}\right)$ where $R_{1}$ and $R_{2}$ are the two principal radii of curvature of the surface at any point. In Fig. 7a, the surface of the dendrite was coloured according to the local values of the mean curvature $H$ of the solid surface reported in Fig. 7b. Red colour corresponds to large positive values of $H$. As shown in Fig. 7a, the interfaces with large positive mean curvature correspond to the smallest tips of the dendrite arms which are observed to dissolve by remelting. With increased solidification, e.g. from 0.19 to 0.65 of solid fraction, the fraction of interfaces with large mean curvature is decreased. The $H$ distribution in Fig. $7 \mathrm{~b}$ then becomes narrower and the predominant colour on the dendrite in Fig. 7a corresponds to an almost zero value of the mean curvature. When the solid fraction exceeds 0.52 , the interfaces with large curvature have disappeared, i.e. the size differences that exist initially between adjacent arms are reduced, and the remelting mechanism, which is driven by the minimization of surface energy due to the Gibbs-Thomson effect, is no longer favoured. In addition, when adjacent arms have grown to such an 
extent that they come into contact with each other, they are observed to coalesce at their tips, as shown in Fig. 6. As suggested by Li [9], coalescence is more probable at these high solid fractions because it does not depend on a difference in tip radii between neighbouring arms.

As pointed out by Alkemper and Voorhees [18], the mean curvature is not sufficient to fully characterize the evolution of the microstructure with time. One reason is that saddle-shaped interfaces at the root of dendrite arms could have a zero mean curvature. Thus, they would be considered inactive with respect to coarsening, which is contradicted by the general observation of the microstructure evolution [18]. The distribution of the Gauss curvature, which is defined as $K=1 / R_{1} \times 1 / R_{2}$, is plotted in Fig. $7 \mathrm{~d}$. The roots of the dendrite arms with saddle-shaped surfaces in Fig. 7c have negative Gauss curvature while the arm tips have positive curvature. Clearly, the $K$ distribution becomes narrower as the saddle-shaped surfaces, i.e. the roots between adjacent arms, disappear due to solidification growth, coalescence of dendrite arms and dissolution of smaller arms.

In order to compare the microstructural evolution on the local scale to the global evolution in the sample volume, the specific solid-liquid interface area, $S_{\mathrm{v}}$ (shown in Fig. 5), was normalized by the volume of solid. $S_{\mathrm{v}}$ was measured both for the single dendrite and for a larger volume element (of $0.3 \mathrm{~mm}^{3}$ ) that contains this dendrite and many others since it corresponds to a solid volume which is about 10 times larger that the dendrite itself.

Fig. 8 shows that the evolution of $S_{\mathrm{v}}$ on the scale of the dendrite is similar to the evolution on the sample scale. Thus, the mechanisms that act on this dendrite are likely to be representative of the mechanisms that act upon a larger volume element. A difference is visible between the dendrite and the sample scale for low solid fractions when the error of measurement is at its highest due to a weak

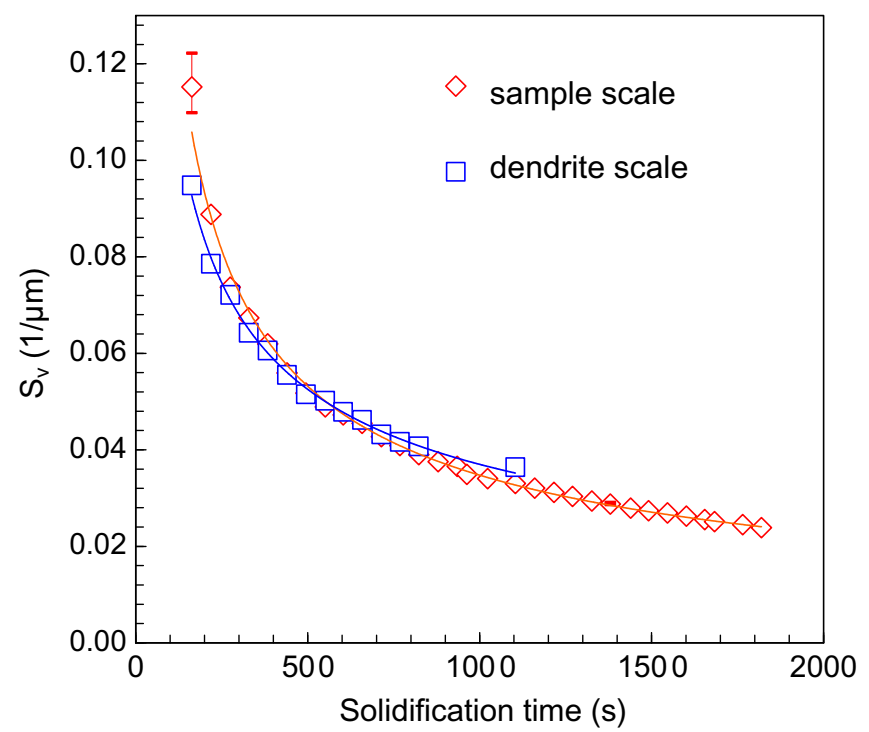

Fig. 8. Variation with time of the specific solid-liquid interface area, $S_{\mathrm{v}}$, on the sample scale and on a local scale. contrast between the solid and the liquid phase. A difference is also observed at the highest solid fractions $\left(g_{\mathrm{s}} \approx 0.76\right)$ when many contacts between adjacent dendrites had to be cut to allow visualization of a single dendrite.

The dendrite was then split in two to allow distinct analysis of the arms that follow model A on the right-hand side of the dendrite in Fig. 6 and of the arms that follow a coalescence mechanism that resembles model $\mathrm{B}$ on the lefthand side of the dendrite in Fig. 6. For the sake of simplicity, the right-hand side and left-hand side of the dendrite will be called "side A" and "side B", respectively.

The specific surface areas measured on side B is plotted in Fig. 9. Its evolution with time is close to the evolution of $S_{\mathrm{v}}$ on the sample scale. The time exponents of the power-law fits are very close, but the $S_{\mathrm{v}}$ values for side B are lower than at the dendrite or sample scale. To the best of our knowledge, there is no appropriate model in the literature that represents the coalescence mechanism observed on side B. Neither the model B proposed by Chen and Kattamis [4] nor the coalescence model proposed by Mortensen in Fig. 1c [6] properly represents the mechanism that was observed to act upon the solidifying dendrite. Because the time evolution of the specific surface area for side B does not differ markedly from the evolution on the sample scale, it seems difficult to determine the influence of this mechanism during solidification from the only characterization of $S_{\mathrm{v}}$. Thus, to better assess the evolution on the local scale, the size evolution of the dendrite arms with solidification time was investigated. The positions of the tips and roots of the dendrite arms were determined as it is easier and more accurate than to measure the tip radius of a dendrite arm.

The coalescence mechanism identified on side B implies a progressive filling of the space between the arms. Thus, the positions of the tips, $L$, and roots, $l$, of the dendrite arm relative to the initial configuration $\left(L_{0}\right.$ and $l_{0}$, respectively) at a solidification time of $162 \mathrm{~s}$, are plotted with

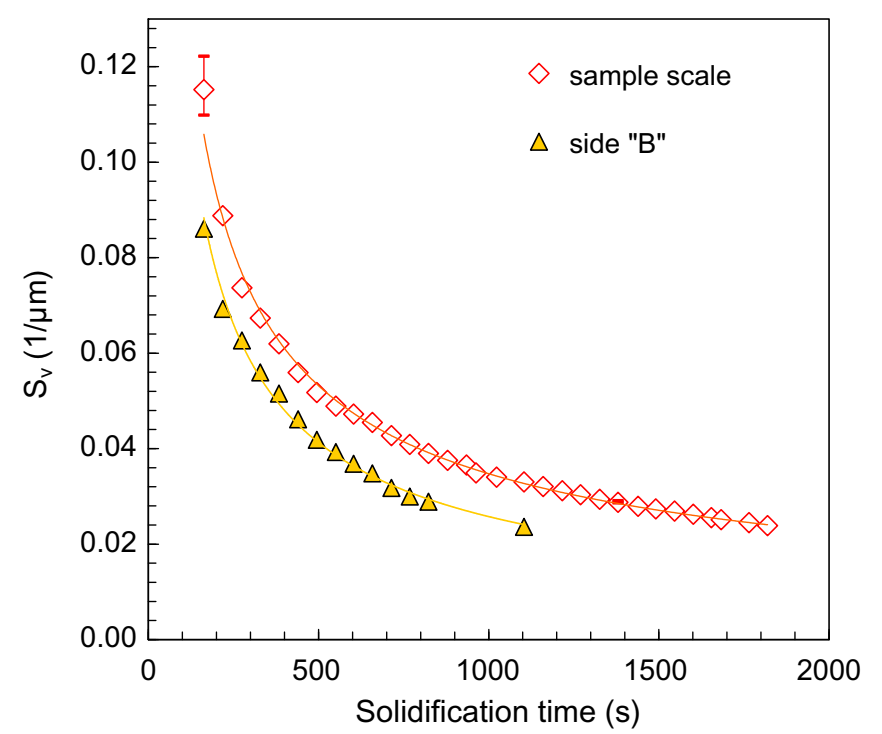

Fig. 9. Variation with time of the specific solid-liquid interface area, $S_{\mathrm{v}}$, on the side arms that follow model B. 

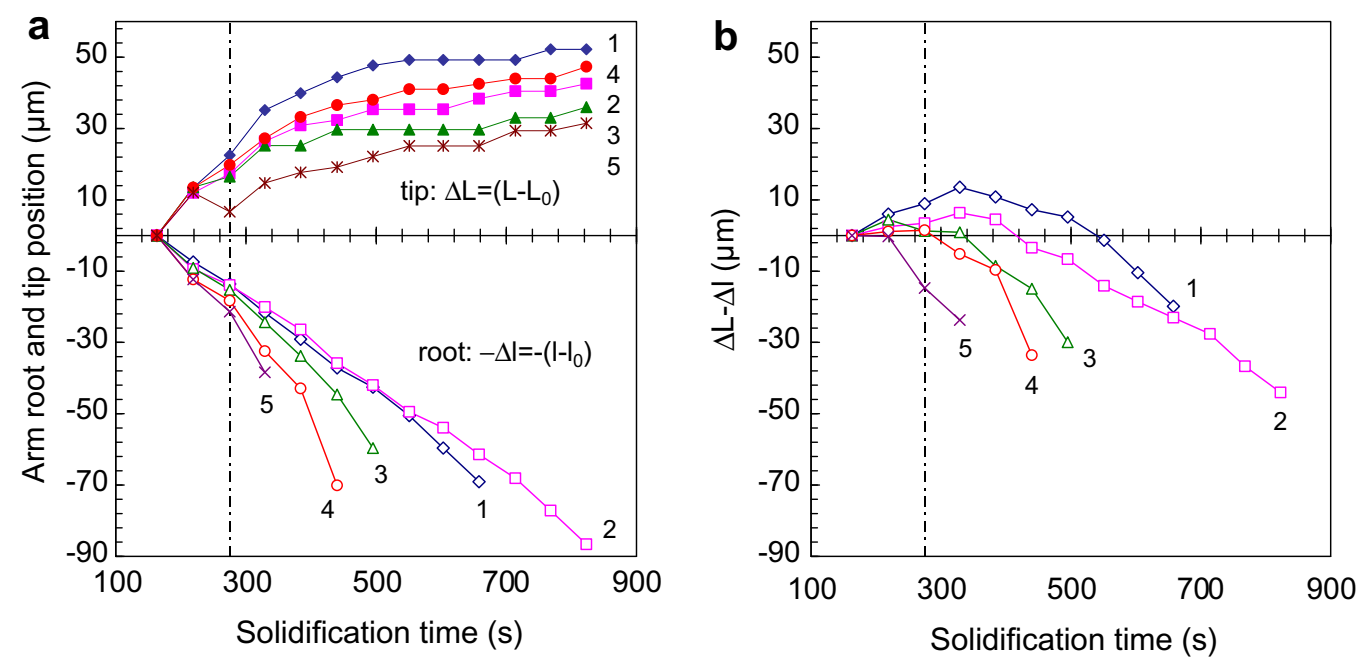

Fig. 10. Variation with time of (a) the relative positions of the dendrite arms tips $(\Delta L)$ and roots $(\Delta l)$ that follow model B (to facilitate comparison with tips, the positions of the roots are plotted along a negative $y$-axis even though $l$ increases) and (b) the difference $(\Delta L-\Delta l)$ between growth of the tips and filling of the roots.

respect to time in Fig. 10a (where the root below the corresponding arm tip is given the same label as the arm). The positions of tips and roots are growing in the same direction, i.e. towards the left-hand side in Fig. 6, which corresponds to an increase in length. However, the root position was plotted with a negative ordinate to easily distinguish root and tip variations. Before $275 \mathrm{~s}$, the increase in absolute values of the positions of the arm tips and roots can result from solidification growth only, i.e. without any coalescence mechanism. The difference between the growth of the arm tips, $\Delta L$, and the filling of the space at the root of the arms, $\Delta l$, is plotted in Fig. 10b. It shows that initially the tips are growing faster than the roots $(\Delta L-\Delta l>0$ in Fig. 10b), which could not be explained by coalescence. The solidification growth seems non-uniform and mostly affects the position of the arm tips. After a short time period when the increase in the length of the dendrite arm due to solidification compensates the filling of the arm roots ( $\Delta L-\Delta l=0$ in Fig. 10b), the filling of the root becomes the dominant mechanism that controls the arm dimensions or morphology ( $\Delta L-\Delta l<0$ in Fig. 10b). This may result from a coalescence mechanism that begins to operate upon the solidifying dendrite arms. Diffusion of matter from the tips of the growing arms to the root causes a rapid filling of the root due to both solidification growth and coarsening. It is interesting to notice that the root of the dendrite arm adjacent to the smallest arm No 5 decreases faster than root No 2, for example, probably because the diffusion length, i.e. from tip to root, is shorter so that the coarsening is faster.

On the right-hand side of the dendrite in Fig. 6, i.e. side A, the remelting mechanism observed strongly resembles the so-called model A proposed by Kattamis et al. [2-4]. In model $\mathrm{A}$, the growth rate of the radii of the largest arms $(\mathrm{d} R / \mathrm{d} t)$ and the corresponding dissolution rate of the height of the smallest $\operatorname{arms}(\mathrm{d} l / \mathrm{d} t)$ are given by the equations below:

$$
\begin{aligned}
& \left.\frac{\mathrm{d} R}{\mathrm{~d} t}\right|_{\text {coarsening }}=\frac{\sigma T D}{C_{l}(1-k) H m} \frac{2 R-a}{R^{2}(L+R)} \\
& \left.\frac{\mathrm{d} R}{\mathrm{~d} t}\right|_{\text {solidification }}=\frac{1}{\pi\left(R^{2}+R L\right)} \frac{\rho_{s}}{\rho_{l}} \frac{\mathrm{d} f_{s}}{\mathrm{~d} t} \\
& \frac{\mathrm{d} l}{\mathrm{~d} t}=-\frac{2 \sigma T D}{C_{l}(1-k) H m}\left(\frac{2}{a}-\frac{1}{R}\right) \frac{1}{a}
\end{aligned}
$$

where $R$ is the radius of the biggest arm that grows with a constant length $L ; l$ is the length of the smallest arm that dissolves with a constant radius $a ; \sigma, D$ and $H$ are the solid-liquid interface energy, the diffusivity of solute in the liquid and the volumetric heat of fusion, respectively; $C_{1}$ and $k$ are the liquid concentration and the partition ratio at a temperature $T ; m$ is the slope of the liquidus line $(-3.394 \mathrm{~K} / \mathrm{wt} . \% \mathrm{Cu}) ; \rho_{\mathrm{s}}$ and $\rho_{1}$ are the solid and liquid densities; and $f_{\mathrm{s}}$ is the mass solid fraction.

Kattamis assumes that solidification growth is limited to the growing arm, which does fit the preliminary qualitative observations in Fig. 6. His model can also be used to calculate the variation of the specific surface area with time:

$S_{\mathrm{v}}=\frac{6\left(R L+R^{2}+a l+a^{2}\right)}{3 R^{2} L+2 R^{3}+3 a^{2} l+2 a^{3}}$

The Kattamis model was applied to the side A of the dendrite under study. The values of $\sigma, D$ and $H$ were taken from Ref. [2] for an Al-Cu alloy. The variation of $C_{1}$ with temperature was estimated from the phase diagram. The liquid and solid densities were assumed to vary with temperature, as reported in Ref. [14]. The solid fraction is calculated with the Scheil equation. The dimensions of the dendrite arms were measured on side $\mathrm{A}$ of the dendrite for the arms labelled from 7 to 17 in Fig. 6 at 163 s of solidification time. The average values for the radius, $a$, and for the initial length, $l_{0}$, at $163 \mathrm{~s}$ of solidification time of the smallest arm are 14 and $61 \mu \mathrm{m}$, respectively. The average length of the biggest arm, $L$, is $183 \mu \mathrm{m}$. The variation of 

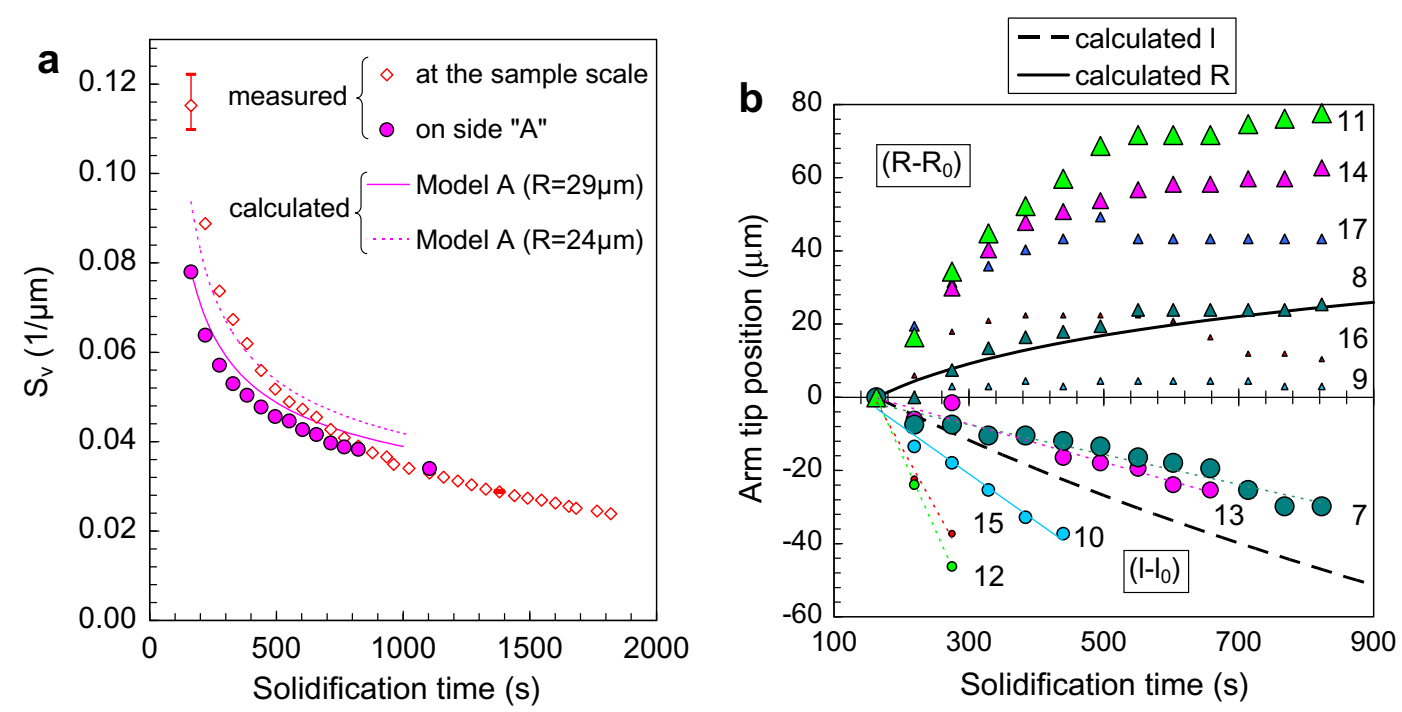

Fig. 11. Variation with time of (a) the measured and computed specific solid-liquid interface area, $S_{\mathrm{v}}$, for the dendrite arms that follow model A and (b) the dimensions of these arms.

the radius of the biggest arm, $R$, and of the length of the smallest arm, $l$, with time was calculated using Eq. (2). Eq. (3) was then used with this set of parameters, using two different values for the initial average radius of the growing arm, $R_{0}$, i.e. 24 and $29 \mu \mathrm{m}$, which are representative of the true shape of the dendrite arm. In fact, Kattamis assumes that arms have a cylindrical shape while the arms observed in this study are tear-shaped. The calculated curves are shown in Fig. 11a, together with the measured evolution of $S_{\mathrm{v}}$ for side A of the dendrite. The calculated curves are in the same order of magnitude and show the same evolution with time as the measured curve. However, the evolution of $S_{\mathrm{v}}$ on side A slightly differs from the evolution of $S_{\mathrm{v}}$ at the sample scale: the time exponent of the power-law fit is -0.4 for side A, while it is -0.6 at the sample scale. This might be ascribed to the major influence of one coarsening mechanism on side A whereas, on a larger scale, other mechanisms may operate and influence the variation of $S_{\mathrm{v}}$ with time.

As previously done with side $\mathrm{B}$, a careful analysis of the evolution of the sizes of the dendrite arms with solidification time was performed on side A to quantitatively assess the influence of remelting on the microstructure. The lengths, $L$, of the growing arms and the radii, $a$, of the shrinking arms are assumed to be constant, as in Kattamis model. The measured positions plotted in Fig. $11 \mathrm{~b}$ thus correspond to the radii, $R$, and the length, $l$, of the growing arms (triangular symbols) and shrinking arms (circular symbols), respectively. Dendrite arms are labelled with the numbers shown in Fig. 6. Fig. 11b reveals that the smaller the initial size of the shrinking arm, the sooner it is dissolved. Accordingly, the larger the growing arm, the faster and larger its radius increases until it reaches a constant size. As soon as the dendrite arm impinges on another adjacent arm, it cannot become any larger and its radius stabilizes. The smallest arms among the growing arms, i.e. labels 16 and 9 , have their evolution reversed when they begin to dissolve to the benefit of bigger arms because all the other smaller arms in the vicinity have already remelted. Using the set of Eq. (2) proposed by Kattamis et al., the relative evolutions of $R$ and $l$ were computed using the average dimensions presented above. The computed curves, plotted in plain and dashed lines in Fig. $11 \mathrm{~b}$, are consistent with the measured evolutions of individual arms.

The remelting mechanism observed for side $\mathrm{A}$ is therefore quantitatively well described by model A both for individual arms on a dendrite, although the true morphology of the dendrite arms, which are tear-shaped and not cylindrical, is not taken into account, and on a global scale.

\section{Conclusions}

Using synchrotron fast X-ray microtomography, an experimental set-up was developed to study in situ and in real-time the microstructural evolution of an $\mathrm{Al}-\mathrm{Cu}$ alloy during solidification at a constant cooling rate of $3 \mathrm{~K} \mathrm{~min}^{-1}$. The evolution of the dendritic microstructure with solidification time was fully characterized in a volume of analysis of $0.3 \mathrm{~mm}^{3}$; the solid fraction, the specific surface area of the solid-liquid interface and the distribution of local curvature were measured.

In addition to solidification growth, at least two coarsening mechanisms were observed to act upon the dendrite arms. These mechanisms were analysed in detail, both qualitatively and quantitatively, on the scale of a representative dendrite. The first mechanism that occurs with solidification time involves remelting of a small secondary dendrite arm to the benefit of larger adjacent arms. Although this mechanism implies large evolutions at the scale of the dendrite arms, it was shown to correlate well with the model A proposed by Chen and Kattamis [4], 
which proves the robustness of the model. Another coarsening mechanism, which seems to dominate at high solid fractions, is coalescence of adjacent dendrite arms. This leads to the progressive filling of the space between dendrite arms with coalescence of the adjacent arms near their tips.

The observed mechanism does not correspond to any known model, as no coalescence model has been proposed for solidification conditions to the best of the authors' knowledge. During solidification, the coalescence mechanism that is observed is a combination of the coalescence mechanism by Mortensen [6] and the model B proposed by Chen and Kattamis [4]. Furthermore, in the present study, remelting and coalescence are found to coexist during solidification of an $\mathrm{Al}-\mathrm{Cu}$ alloy. Consequently, any model that involves the operation of model A followed by a pseudo-model $\mathrm{B}$ poorly represents the experimental observations and more sophisticated models are required.

The observations reported in this study were carried out at a cooling rate of $3 \mathrm{~K} \mathrm{~min}^{-1}$. It might be interesting to conduct the same solidification experiment at different cooling rates to study the influence of this parameter on the coarsening mechanisms observed. At higher cooling rates, coarsening mechanisms have probably less influence on the dendrite morphology. However, it would be necessary to further reduce the acquisition time of a tomographic scan in order to carry out in situ observations of dendritic solidification at higher cooling rates.

\section{Acknowledgements}

This work was carried out in the framework of the project ANR-05-BLAN-0286-01 "TOMOSOLIDAL", supported by the "Agence Nationale de la Recherche", which is gratefully acknowledged. The authors wish to thank all staff members of the ID19 beam line of ESRF Grenoble for their technical support. They are also grateful to Samuel Forest from the Ecole des Mines de Paris for providing the furnace.

\section{References}

[1] Reeves JJ, Kattamis TZ. Scr Metall 1971;5:223.

[2] Whisler NJ, Kattamis TZ. J Cryst Growth 1972;15:20.

[3] Peterson PW, Kattamis TZ, Giamei AF. Met Trans A 1980;11A:1059.

[4] Chen M, Kattamis TZ. Mater Sci Eng A 1998;A247:239.

[5] Young KP, Kirkwood DH. Met Trans A 1975;6A:197.

[6] Mortensen A. Met Trans A 1989;20A:247.

[7] Reinhart G, Mangelinck-Noël N, Nguyen-Thi H, Schenk T, Gastaldi J, Billia B, et al. Mater Sci Eng A 2005;A413-414:384.

[8] Arnberg L, Mathiesen RH. JOM 2007;59:20.

[9] Li B, Brody HD, Kazimirov A. Metall Mater Trans A 2007;38A:599.

[10] Ruvalcaba D, Mathiesen RH, Eskin DG, Arnberg L, Katgerman L. Acta Mater 2007;55:4287.

[11] Cloetens P, Ludwig W, Guigay J-P, Baruchel J, Schlenker M, Van Dyck P. In: Baruchel J, Buffiere J-Y, Maire E, Merle P, Peix G, editors. X-ray tomography in material science. Paris: HERMES Science Publications; 2000. p. 29-43.

[12] Ludwig O, DiMichiel M, Salvo L, Suéry M, Falus P. Metall Mater Trans A 2005;36:1515.

[13] Limodin N, Salvo L, Suery M, DiMichiel M. Acta Mater 2007;55:3177.

[14] Ganesan S, Poirier DR. Met Trans A 1987;18A:721.

[15] Lorensen WE, Cline HE. Comput Graph (ACM) 1987;21:163.

[16] Rath BB. In: Aaronson HI, Laughlin DE, Sekerka RF, Wayman CM, editors. Solid-solid phase transformations. Warrendale, PA: TMSAIME; 1982. p. 1097-103.

[17] Cahn JW. Trans Metall Soc AIME 1967;239:610.

[18] Alkemper J, Voorhees PW. Acta Mater 2001;49:897. 